\title{
Modélisation sémantique, sens différentiel et genre
}

\author{
Fabienne Baider \\ Université de Chypre \\ fabienne@ucy.ac.cy \\ Evelyne Jacquey \\ ATILF \\ ejacquey@atilf.fr
}

\section{Asymétrie discursive et sémantique}

De nombreux travaux sur la langue française ont identifié une asymétrie discursive entre :

- d'une part la conceptualisation en langue et en discours de l'être féminin et de l'être masculin dans la langue française (Yaguello 1989 ; Houdebine-Gravaud 2000);

- d'autre part le traitement des notions « être féminin » et « être masculin » en discours métalinguistique (Beaujot et Lehman 1979 ; Benhamou 1986 ; Khaznadar 2000 ; Michard 2002 ; Scullen 2005).

Outre les observations faites en discours sur des exemples impliquant les lexèmes homme et femme (section 1.1), nous verrons en (1.2) et (1.3) que les descriptions conceptuelles et lexicographiques de homme et de femme comportent elles aussi des asymétries concordantes.

\subsection{Observations en discours}

Plusieurs études menées sur le paradigme féminin ont mis au jour un phénomène discursif récurrent : la dévalorisation de nombreux termes désignant les femmes, des termes féminisés, et des termes faisant référence à des activités dites féminines; ceux-ci sont minorants, minimisants ou péjorants (Françoise Armengaud, 1999 : 3). Cette dévalorisation apparaît notamment dans les paires minimales à sens différentiel du type N+Adj ou Adj+N. Ce phénomène n'est pas nouveau et a été étudié par Lakoff (1975), Schultz (1975) et Cameron (1990) dans la langue anglaise. Il a été appelé semantic derogation (Schultz, 1975), semantic degeneration (Miller et Swift, 1976) et semantic polarization (Eakins et Eakins, 1978), mais n'a jamais été formalisé. Dans la suite, nous appellerons ces paires minimales à sens différentiel, syntagmes à sens différentiel.

En français, nombre de ces syntagmes sont considérés comme des expressions figées, mais ils ne le sont pas nécessairement tous, ainsi femme libre / homme libre :

(1) femme publique / homme public

(2) femme facile / homme facile

(3) grande femme / grand homme

De plus, lorsqu'ils le sont, on constate une forte dissymétrie interprétative, ainsi en (1), la prostituée avec femme et « homme qui exerce des fonctions officielles » avec homme d'après le TLFi ${ }^{1}$. On note aussi une dissymétrie relativement au figement lui-même, (2-3), en plus de la dissymétrie interprétative.

Lorsqu'il y a dissymétrie interprétative, les auteurs notent notamment une connotation sexuelle très fréquente avec le nom femme «femme dont on obtient sans peine les faveurs » pour femme facile d'après le TLFi, ou encore une connotation relativement aux moeurs sociales liées au fait que la femme est la partenaire sexuelle de l'homme. C'est le cas avec plusieurs adjectifs comme facile, galant, léger, honnête, mais aussi d'autres adjectifs qui appartiennent à des champs sémantiques proches des premiers, sage et sérieux s'appariant à honnête, ou encore libre à léger et facile. En effet, en corpus (FRANTEXT), les trois 
occurrences de femme sage décrivent la conduite sexuelle de l'intéressée et ce syntagme est alors synonyme de « sensée dans sa conduite sexuelle ». En comparaison, 87\% des occurrences de homme sage ont le sens d'un « homme de bon conseil ». La recherche dans ce corpus confirme aussi la restriction de l'acception sexuelle de l'adjectif sérieux au référent féminin. De même, l'emploi de l'adjectif libre dans ce type de configuration est aussi le plus souvent restreint à la conduite licencieuse de la femme plutôt qu'à celle de l'homme. Tous ces adjectifs partagent la notion «absence ou présence d'obstacle à une relation » (Baider et Brousseau 1998). Comme le montre un examen en corpus, cette notion semble avoir plus souvent pour focus le comportement sexuel lors de la référence à l'être féminin que lors de la référence à l'être masculin. Cela est aussi vrai dans des bases de données telles que EuroWordNet.

\subsection{Observations de l'ontologie sémantique EuroWordNet}

EuroWordNet est une base de données lexicales multilingues représentant de manière hiérarchique l'ensemble des sens des noms et des verbes pour plusieurs langues européennes. Cette base multilingue obéit aux mêmes principes que WordNet exclusivement consacrée à l'anglais (Fellbaum 1998). Elle est à ce titre employée par les traducteurs et les linguistes. Dans cette base, chaque « sens » est représenté par un « synset». Un synset est un ensemble de sens quasi-synonymes entre eux. Ainsi par exemple, le synset \{être humain:2; homme:4\} correspond à un certain sens, qualifié par une glose ou définition, qui est partagé par le second sens de être humain, noté \{être humain:1\}, et le quatrième sens de homme, noté \{homme:4\}. Chaque synset est de plus situé dans la hiérarchie selon la relation hyperonymie / hyponymie. En outre, un synset peut entretenir entre autres des relations de méronymie avec d'autres synsets de la base. Nous reproduisons ci-dessous les représentations de homme et de femme dans cette base (Fig 1).

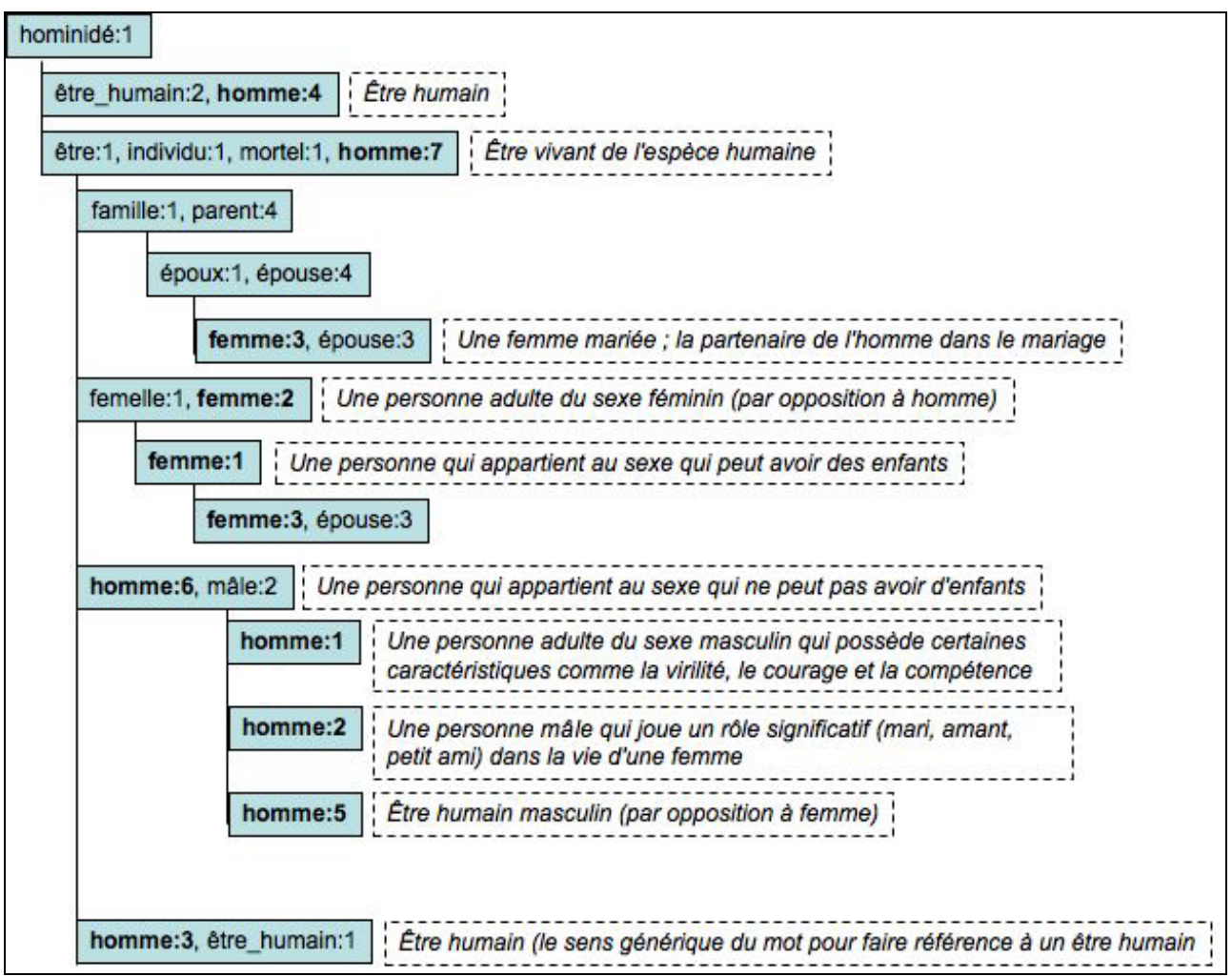

Fig 1 : Représentation de homme et femme dans EuroWordNet 
Comme le montre cette représentation, si le synset \{hominidé:1\} domine logiquement la hiérarchie, le caractère générique du mot homme dans le sens 4 (être humain) est renforcé par deux autres sens : le sens 7 (être vivant de l'espèce humaine) qui domine à la fois tous les autres sens de homme et de femme et le sens 3 (sens générique du mot pour faire référence à un être humain). Sur ce point, on peut donc conclure que trois sens du mot homme sont relatifs à la notion d'être humain et pas spécifiquement à celle d'être masculin. Comparativement, on notera qu'aucun sens du mot femme ne fait référence à cette notion d'être humain, ce entre en résonance avec le fait que le mot femme n'est pas utilisé à cette fin en discours. Dans ces représentations hiérarchiques, l'usage du masculin générique masque donc le trait humain contenu dans le mot femme. L'asymétrie entre les deux notions semble impliquer que le mot femme serait plus un hyponyme de homme que son co-hyponyme. Cette analyse se voit renforcée par les gloses des synset faisant intervenir les sens 3, 4 et 7 de homme ${ }^{2}$.

Enfin, la lecture de l'ensemble des gloses des synsets contenant l'un des sens de homme ou de femme conduit à deux observations : (1) la survie d'un certain nombre de stéréotypes culturels et (2) le caractère strictement fonctionnel et/ou limitatif des gloses décrivant le sens de femme. En effet, si l'on isole les trois sens génériques de homme, il reste quatre sens relevant du paradigme d'être masculin à comparer avec les trois sens de femme relevant exclusivement du paradigme d'être féminin. Un premier sousensemble dans ces sept sens à comparer montre une quasi-équivalence de traitement entre femme et homme: les sens \{homme:5\} et \{homme:6\} sont quasiment complémentaires de $\{$ femme:2\} et \{femme:1\} respectivement. En revanche, des différences importantes apparaissent avec les deux sens restant de homme, \{homme:1\} «une personne adulte du sexe masculin qui possède certaines caractéristiques comme la virilité, le courage et la compétence », \{homme:2\} « une personne mâle adulte qui joue un rôle significatif (mari, amant, petit ami) dans la vie d'une femme », et \{femme:3\}, "une femme mariée ; la partenaire de l'homme dans le mariage ». Premièrement, si \{femme:3\} et \{homme:2\} semblent assez proches, on notera que les comportements possibles sont plus divers pour l'homme que pour la femme qui semble cantonnée au mariage. Deuxièmement, on peut remarquer d'une part que l'homme est agent dans la glose, mais pas la femme, et d'autre part, qu'elle ne semble pas «jouer un rôle significatif dans la vie d'un homme » comme c'est le cas pour \{homme:2\}. Enfin, le cas de \{homme:1\} illustre particulièrement bien le phénomène de dévalorisation du paradigme féminin, ici dans le cas d'une description métalinguistique. Ce sens décrit un stéréotype bien connu associé au paradigme masculin ayant pour caractéristique «la virilité, le courage et la compétence ». Si l'on compare aux descriptions de femme, la même conclusion émerge : le paradigme féminin est amputé des caractéristiques que sont le courage et la compétence. Cette conclusion peut être tirée de deux manières sur la base des gloses et de l'héritage :

- soit \{homme: 1$\}$ n'a pas d'équivalent dans le paradigme féminin car ce sens n'a pas de co-hyponyme direct faisant intervenir femme et ni de co-hyponyme indirect. Si l'on recherche une co-hyponymie indirecte en passant par l'équivalence entre le \{femme:1\} et le \{homme:6\} relativement à avoir ou ne pas avoir l'aptitude à avoir des enfants, alors l'hyponyme de \{femme:1\}, femme:3\}, la femme mariée, ne peut pas être comparé à l'hyponyme de \{homme:6\}, l'homme viril, courageux et compétent.

- soit \{homme:1\} peut être comparé à \{femme:2\} sur la base d'une similitude de forme entre les deux gloses, «une personne adulte du sexe féminin », respectivement «masculin». Alors à nouveau, cette comparaison conduit à l'idée que la femme ne peut être caractérisée par le courage et la compétence, ou toute autre référence à la personnalité ou au comportement social.

On le voit donc bien ici, les constatations de dévalorisation ou de restriction de l'être féminin en discours se voient confirmées dans les descriptions métalinguistiques fournies par la base EuroWordNet.

\subsection{Discours lexicographique}

Dans le TLFi, l'extraction du contenu sémantique des mots homme et femme confirme une telle asymétrie entre les deux notions. Nous avons basé l'extraction des données et l'analyse de celles-ci en prenant comme base l'approche sémantique décrite en sémantique textuelle, entre autres (Rastier 1987). Toute information pertinente a été relevée, en particulier les verbes, adjectifs et les noms trouvés dans les 
définitions des deux mots. Ce premier corpus compte 86 mots qui sont ensuite traités comme des traits sémantiques. Un test de fréquence permet de mettre en évidence les traits les plus saillants développé au sein de l'ATILF par Mick Grzesitchak (2008). Enfin, tenant compte de la richesse lexicale des traits dans la mesure où ceux-ci sont issus du TLFi, certains d'entre eux ont été regroupés sur la base de critères morphologiques et/ou sémantiques afin de rendre les différences de saillance plus significatives. Le tableau ci-dessous synthétise les résultats de cette analyse.

\begin{tabular}{|c|c|c|}
\hline & $\begin{array}{l}\text { homme } 2= \\
\text { ettre masculin }\end{array}$ & Femme \\
\hline $\begin{array}{l}\text { /social/, /origine/, /époque/, /catégorie sociale/, } \\
\text { /activité/, /supérieur/, /hiérarchie/, /féodale/, } \\
\text { /militaire/, /civile/, /dépendant/, /sous autorité/, } \\
\text { /soldat/, /équipe/, /entreprise/ }\end{array}$ & 13 & 4 \\
\hline /mâle/, /masculin/, /homme/ & 7 & 2 \\
\hline /être/ /individu/ & 3 & 11 \\
\hline /adulte/, /maturité/, /âge/, /nubile/, /en éveil/ & 7 & 5 \\
\hline /sexuel/, /sexe/, /sexuellement/, /sexualité/, /sexué/ & 2 & 13 \\
\hline /féminin/, /femme/, /féminité/ & 0 & 9 \\
\hline /anatomie/, /physique/, /physiologie/, /corps/ & 0 & 6 \\
\hline
\end{tabular}

Fig 2 : Résultat de l'analyse en traits sémantiques des définitions de homme et femme dans le TLFi

Une telle analyse conduit à la conclusion que les traits les plus saillants sont, par ordre décroissant : pour homme2, équivalent de la notion d'être masculin dissociée de celle d'être humain, [/être_social/:13, /adulte/:7, /masculin/:7], et pour femme, [/être_sexué/:13, /individu/:11, /féminin/:9, /physique/:6]. Autrement dit, le nom homme, lorsqu'il entre dans le paradigme de l'être masculin, est avant tout décrit comme un être social. Comparativement, le nom femme est principalement décrit comme un être sexué. Même si la polysémie de homme "être humain générique » et "être humain mâle » et celle de femme « être humain femelle » et " épouse, partenaire » peut influencer ces résultats, ils corroborent ce qui a été précédemment attesté en discours, dans EuroWordNet et dans d'autres langues qui ne présentent pas la même polysémie, ainsi en anglais wife et woman font la différence entre les deux sens de femme. Il semble donc que, les paradigmes masculin et féminin reçoivent une description bien différenciée dans ce contexte lexicographique. De fait, selon les recherches de Olsen $(1991)^{3}$ sur la base de données FRANTEXT, le terme co-occurrent le plus fréquent avec le nom sexe, toutes époques confondues, est le nom femme(s). Cette co-occurrence n'est pas vraie pour le mot homme. Cette co-occurrence textuelle du nom femme et du nom sexe peut supposer une association conceptuelle de " femme " et de "sexe ".

Cette représentation confirme les résultats en discours (dévalorisation et restriction pour l'être féminin) et dans les hiérarchies sémantiques (le mot femme étant considéré plus comme un hyponyme de homme qu'un co-hyponyme). Dans la suite, nous nous concentrons sur le cas des paires minimales de la forme $\mathrm{N}+$ Adj ou Adj+N. Plusieurs observations et analyses permettront alors de mieux qualifier le phénomène constaté et d'établir une problématique de formalisation suffisamment bien définie pour en proposer une mise en oeuvre dans la dernière section. 


\section{Syntagmes $\mathbf{N}+$ Adj ou Adj+N}

Comme cela a été mentionné, dans ces syntagmes, la relation entre le nom et l'adjectif ne s'interprète pas de la même manière selon que le nom est réalisé par homme ou femme. Les syntagmes de cette forme ont été choisis car leur analyse pourrait mener à une explication plus générale de l'asymétrie observée en discours et dans les descriptions métalinguistiques.

\subsection{La différence est-elle fondée sur des contraintes distributionnelles ?}

Concernant ces syntagmes à sens différentiel, seraient-ce les contextes dans lesquels ils apparaissent, ou bien les positions relatives du nom et de l'adjectif, qui permettraient d'expliquer les interprétations constatées? En effet, la position de grand dans grand homme ou homme grand conditionne bien une différence interprétative. On notera cependant que cela n'est pas vrai avec le nom femme $e^{4}$. De plus, quel que soit le contexte, l'alternative possible que l'on connaît pour [grand +homme], [grande + dame] et [grand + monsieur] n'existe pratiquement jamais en corpus (Base FRANTEXT ou recherches GOOGLE) pour [grande + femme]. Le sens de ces syntagmes semble ici davantage influencé par le nom femme que par les contextes ou la position de grand. De même, lorsque l'adjectif est uniquement postposé, les syntagmes homme loyal et femme loyale présentent une différence de sens, alors que la relation d'intersection unit les deux unités de la même manière. La même analyse pourrait être faite avec l'adjectif antéposé.

L'origine de la différence dans le cas de ces syntagmes étude doit donc se trouver plutôt dans la sémantique du nom et de l'adjectif, dans la relation nom-adjectif, et non dans la syntaxe c'est-à-dire dans la position de l'adjectif.

\subsection{Ambiguïté de l'adjectif ?}

Une autre piste d'explication pourrait être que les adjectifs sont sémantiquement ambigus dans ces syntagmes. Comme le souligne cependant (Bouillon 1997), adopter une telle analyse conduirait à une explosion du lexique. Avec l'adjectif honnête par exemple, il faudrait postuler de nombreux sens mis en évidence dans des paires N+Adj très diverses d'après le TLFi par exemple : pour courtier, marchand " exercer un métier sans fraude ni falsification", pour homme, femme "être conforme à une norme sociale (sexuelle pour les femmes) $)^{5} »$, pour homme, gens, «être agréable aux autres », pour prétexte, refus, «action respectant les principes de savoir-vivre et de politesse », pour prix, repas, récompense « objet conforme à l'usage établi » et pour fortune, résultat, culture, livre, condition, naissance, joueur «se trouver dans la moyenne ». Comme le propose le lexique génératif (Pustejovsky 1995 ; Bouillon 1997), la variété interprétative des réalisations d'adjectifs comme honnête, mais aussi rapide ou facile, peut être représentée plus économiquement et plus explicitement par les contenus sémantiques des noms, celui de l'adjectif mais aussi grâce aux mécanismes dits génératifs de cette théorie qui induisent une compositionnalité étendue.

\subsection{Sème afférent ?}

Selon la théorie de Rastier, tout nom est défini par des sèmes (ou traits) inhérents ou définitoires et par des sèmes afférents activés par le contexte ou le cotexte linguistique. Les sèmes afférents, et en particulier les sèmes localement afférents, expriment ce qu'on appelle une "connotation». Ainsi, les sèmes afférents sont des éléments latents "dans la mémoire associative du sujet parlant". La mémoire associative doit jouer dans la construction des archétypes et les construits socio-culturels.

Rastier (1987 : 71) explique la variation sémantique et en particulier celle des adjectifs, par l'interactivité entre adjectif et nom. A partir de l'observation d'occurrences de facile et de difficile, il postule que ces deux adjectifs contiennent le sème /action/. Dans un problème est difficile, le problème est difficile $\grave{a}$ résoudre, résoudre étant l'action sous-entendue dans le nom problème. Ce sème /action/ sera alors réalisé sous la forme d'un sème plus précis et correspondant à une représentation socialement normée. De plus, 
ces adjectifs ne pourront s'associer qu'à des noms comportant une action dans leur sémème. Dans ce cas, lorsque de la composition avec l'adjectif, celui active le sème d'action du sémème du nom.

(4) Un texte difficile/facile = « difficile ou facile à comprendre » [sème afférent =/compréhension/]

(5) Un enfant difficile/facile = « difficile ou facile à éduquer » : [sème afférent = /éducation/]

(6) Une personne/un homme difficile/facile = «un homme difficile ou facile à satisfaire ou à supporter) » [sème afférent =/satisfaire/ ou /supporter/]

Si le nom ne contient pas de sème spécifique précisant l'action, l'association avec l'adjectif n'est pas aisée. Rastier (1987 : 72) donne l'exemple suivant : Un arbre difficile (À faire pousser ? À élaguer ?) afin de montrer que difficile "ne permet pas d'afférence socialement normée" dans ce contexte puisque arbre ne contient pas d'action spécifique. Cependant comme les sèmes afférents relèvent d'autres codifications et dépendent de normes socialisées, voire idiolectales (1987:44), dans des domaines de référence différents la présence d'un sème afférent dans la co-occurrence de arbre et des adjectifs faciledifficile serait possible comme d'ailleurs l'attestent des occurrences trouvées sur Google telles que Un bonzaï facile à vivre, Un arbre facile à entretenir. L'ellipse des verbes (à entretenir, à vivre) ne peut être réalisée que lors d'une fréquence d'emploi élevée de [arbre + facile à entretenir], par exemple. En effet, l'étude diachronique de femme facile le suggère : femme (de vertu) facile étant plus courant au $17 \mathrm{eme}$ siècle que femme facile, l'inverse étant vrai quelques deux cents ans plus tard (Baider 2004) ${ }^{6}$.

Si l'on considère femme difficile, ce syntagme est aussi effectivement compris " une femme difficile (à satisfaire ou à supporter) ", par défaut, même si un contexte spécifique peut déclencher l'interprétation sexuelle (comme c'est le cas aussi pour homme facile) ; en revanche, le syntagme une femme facile est compris comme une femme facile (à séduire) : quel sème afférent inclure dans facile pour expliquer la différence ? Quels mécanismes pourraient rendre compte de choix différents de sèmes afférents selon les contextes?

Si toute relation d'afférence socialement normée doit être inscrite dans le lexique, et cela afin de rendre compte des interprétations distinctives, il faudrait que le mot femme contienne un trait sémantique pour rendre compte des interprétations sexuelles et socialement restreintes. Il existerait un axiome normatif dépendant de normes socialisées selon lequel la femme est "un être à séduire ", ce topos étant aussi largement attesté que celui de "la femme est un être faible" identifié par Rastier. Conséquemment, Rastier propose d'inclure le trait /faiblesse/ en tant que sème afférent dans le sémème de "femme ". L'association de la séduction à l'être féminin pourrait expliquer l'interprétation des syntagmes femme galante, honnête femme ou femme légère; l'axiome normatif serait que si l'adjectif réfère à une relation sociale, celui-ci met en jeu les mœurs et conséquemment la vertu dans le cas du nom femme. Il est entendu que nous pouvons, le plus souvent, revenir au sens par défaut de l'adjectif si le contexte contraint une telle interprétation: dans un texte sur la charge pondérale, il va de soi que le syntagme femme légère ou femme très légère fera référence, comme d'ailleurs homme léger dans ce contexte au poids. Ce qui fait l'objet de ce travail c'est justement la formalisation d'inférences, devenues le sens par défaut.

\subsection{Traitement sémantique différent pour homme et femme ?}

Avant d'entrer plus avant dans la formalisation proposée pour les différentes interprétations de ces syntagmes, examinons quelques éléments qui pourraient invalider l'idée de représenter différemment le contenu sémantique de homme et de femme.

Si des interprétations différentes (péjorées et sexuelles) sont vraies pour les syntagmes à sens différentiel, ces syntagmes ne comprennent que certains adjectifs. Les syntagmes femme intelligente et homme intelligent ne présentent pas de sens différentiel a priori, ni les syntagmes femme difficile et homme difficile. Afin de proposer une explication, on se propose d'étudier ce qui différencie des adjectifs tels que galant, léger, facile, qui ont une interprétation différente lorsqu'ils co-occurrent avec femme, d'adjectifs 
comme intelligent, difficile, qui eu ne varient a priori pas. Littéralement, hors contexte, les adjectifs galant, léger, honnête et facile expriment la civilité (conformité à une norme sociale de comportement).

Ainsi, le premier élément qui semble différencier les deux ensembles d'adjectifs pourrait être ce qu'ils qualifient dans le référent du nom modifié, à savoir le comportement social, social et sexuel pour la femme. De ce point de vue, intelligent ne porte pas préférentiellement sur le comportement social mais plutôt sur les aptitudes intellectuelles.

Reste donc le cas d'adjectifs comme difficile mais aussi impossible. Ces adjectifs, pour leur part, s'attachent au comportement social comme le montre le test de la négation de (Cruse 1986) : cette femme / cet homme est difficile $=($ elle $/$ il $)$ est difficile à vivre / (elle / il) n'a pas un caractère facile. Ces adjectifs, cependant, désignent des propriétés négatives, dans la mesure ou tous deux présentent un obstacle aux relations sociales. Par ailleurs, concernant les adjectifs à sens différentiel galant, léger, honnête et facile, on notera qu'en plus de leur valeur littérale liée au comportement social, celui-ci se trouve particularité lorsqu'il concerne l'être féminin :

- une valeur négative associée au référent féminin a été attestée pour les adjectifs galante, facile, légère dans le cas de femme, ces rapports sociaux sont réduits à la séduction.

- une valeur positive dans le cas de l'adjectif honnête qui dénote un comportement qui contraint le comportement social de femme. On peut paraphraser honnête femme par cette définition: "qui présente un obstacle (à la séduction) ".

Dans ce cas, une hypothèse possible serait que l'interprétation sexuelle et restrictive avec femme ne serait plus l'interprétation par défaut pour des adjectifs tels que difficile, impossible qui désignent déjà des attitudes socialement normées et attendues pour une femme de faire obstacle.

Une autre objection à une traitement sémantique différent de homme et femme pourrait être que d'autres adjectifs appartenant au même champ sémantique que galant, léger, honnête et facile, ainsi les adjectifs chaste, pudique, ne présentent pas de différence de sens lorsqu'ils apparaissent avec homme et femme. Cela peut s'expliquer notamment car chaste et pudique comportent déjà un contenu sémantique ayant trait au comportement sexuel et sexué. Lorsqu'ils sont employés avec homme, leur contenu sémantique enrichit le contenu du nom, produisant ainsi l'interprétation que l'on pourrait attribuer à homme chaste. Dans le cas d'un emploi avec femme, les contenus sémantiques de l'adjectif et du nom sont simplement compatibles avec le socialement normé et aucune modification n'est sensible. Comme le souligne (Rastier 1987), le processus de modification est une interaction entre le nom et l'adjectif : le contenu sémantique du nom modifie celui de l'adjectif tout autant que celui de l'adjectif modifie celui du nom. Ce point de vue est d'ailleurs partagé et mis en oeuvre dans le lexique génératif.

Enfin, le dernier élément que nous aimerions examiner à l'encontre d'un traitement différent de homme et de femme tient au fait que le nom dame ne déclenche pas la même interprétation que femme. Il fonctionne de la même manière que les noms monsieur et homme. Employé avec l'adjectif grand, par exemple le syntagme grande dame peut aussi déclencher une interprétation qualitative. De même, l'adjectif grand en syntagme avec d'autres noms appartenant au paradigme "être féminin" signifie l'excellence dans la fonction dénotée par la classe lexicale : une grande friponne est en effet grande dans sa fonction de friponne et une grande princesse est grande dans sa fonction de princesse. Seul le syntagme grande femme semble résister à l'interprétation qualitative. Pourquoi cette exception? Les deux noms femme et dame se différencient par le seul trait /respect/ contenu dans le mot dame, à cause du rang social que dénote ce mot ou de l'état civil dénoté (mariée). Ainsi Bailly (Dictionnaire des synonymes, 1947) précise que le nom dame est synonyme du nom femme dans la mesure où le mot dame désignait " les femmes mariées" ou "de qualité ". Si l'interprétation qualitative de dame fait référence à son statut social, à sa fonction sociale, quel serait le rôle ou la fonction contenue dans le mot femme pour justifier les interprétations différentes? De même, quel est le trait dans le nom homme et dans le nom femme qui pourrait expliquer une interprétation qualitative pour le nom homme et une interprétation quantitative pour le nom femme? 
En conclusion, les paires minimales de la forme $\mathrm{N}+\mathrm{Adj}$ et $\mathrm{Adj}+\mathrm{N}$ reçoivent des interprétations différentes avec homme et femme dans les conditions suivantes :

- leur fréquence d'usage est élevée, la différence de sens se construisant peu à peu sous l'influence d'éléments qui composent le discours socio-culturel. Ce dernier définit les caractéristiques des référents selon des normes purement culturelles,

- l'adjectif fait référence à la relation sociale et il est positif.

\section{Principes de formalisation}

La théorie du Lexique génératif (LG) de Pustejovsky (1995) offre justement un modèle dynamique de la description sémantique qui semble pouvoir résoudre tout sens contextuel et en particulier celui observé dans les exemples ci-dessus. En effet selon les principes du LG, toute signification contextuelle pourrait être expliquée par ce modèle, tant que cette signification est par défaut la signification vraie dans le contexte donné.

\subsection{Théorie du Lexique Génératif (LG)}

Les interprétations des syntagmes du type $\mathrm{N}+\mathrm{Adj}$, et en particulier l'apparente polysémie des adjectifs facile, galant, etc., doivent ainsi pouvoir être expliquées dans ce modèle tout en évitant la liste de sens contextuels dans une entrée lexicographique, solution ad hoc. La variation que nous avons observée pourrait être expliquée dans ce cadre par :

- les hiérarchies de types avec des relations d'héritage ;

- la structure de qualia qui résume les différentes perspectives d'entité décrites inspirée des causes aristotéliciennes, rappelées dans (Bouillon 1997), ces perspectives correspondent à quatre facettes ou rôles dans LG (formel, ce qui distingue le référent dans un ensemble; constitutif, les éléments constitutifs du référent ou le tout dont il fait partie ; télique, l'usage habituel ou la finalité du référent ; agentif, les conditions nécessaires ou le processus de création du référent). Par exemple, la structure du qualia du nom livre, selon Pustejovsky (1995: 86) rend compte de certaines caractéristiques des référents qu'on appelle des livres : c'est un objet physique et informationnel, il est constitué de pages, d'une couverture, sa fonction est avant tout d'être lu et il résulte du processus d'écriture :

[livre [QUALIA [Formel='objet_phys et info' ; Constitutif='pages, couverture qui contiennent de l'information' ; Agentif='objet physique et informationnel écrit par quelqu'un' ; Télique='objet physique et informationnel destiné à être lu]]]

Une partie importante des informations nécessaires pour l'interprétation des mots en contexte sont donc disponibles et permettent d'expliquer les interprétations contextuelles. Ainsi quand nous lisons la phrase ce livre est difficile, si nous inférons que le livre est difficile à lire, nous faisons référence à la fonction télique du mot livre. Si nous inférons qu'il est difficile à écrire, nous faisons référence à la fonction agentive de ce mot. De même dans la phrase ce livre est vraiment long (Pustejovsky, 1993, p90), nous pouvons comprendre selon le contexte soit que le commentaire se rapporte à la longueur de l'entité physique et l'adjectif fait alors référence aux propriétés formelles du mot livre, soit qu'il se rapporte $\underline{\text { au }}$ temps de lecture, l'adjectif fait alors référence à la fonction télique du mot livre. De même, la sélection dans le rôle constitutif d'un élément différent explique aussi la différence de sens dans les exemples suivants: Mon beau livre est tout égratigné vs Mon beau livre est tout chiffonné dans le premier la couverture serait sélectionnée et dans le deuxième les pages.

Cette inter-activité n'est pas faite au hasard. Trois mécanismes possibles pour sélectionner le rôle approprié sont possibles : le liage sélectif, la co-composition ou la coercion de types.

- Le liage sélectif est un mécanisme qui permet de sélectionner, dans certaines conditions, un autre rôle que le rôle formel dans la structure de qualia. Dans le cas du syntagme une dactylographe rapide, 
l'adjectif rapide devant s'appliquer à une action et la représentation de dactylographe ayant pour rôle télique 'action de taper à la machine', le contenu sémantique du syntagme 'une dactylographe qui tape rapidement à la machine' est obtenu par liage sélectif appliqué au rôle télique du nom sous l'influence des contraintes de type apportée par l'adjectif.

- La coercion de types permet de modifier le type sémantique d'une structure qualia. Ainsi pour construire la représentation de livre déchiré, sachant que déchiré modifie préférentiellement un objet strictement physique et que le rôle formel de livre est instancié par un paramètre physique et informationnel, le mécanisme de coercion de type permet de construire le type de la structure de qualia du syntagme, le type physique uniquement, et de sélectionner dans la structure de qualia du nom tout ce qui touche à l'aspect physique du référent livre.

- La co-composition décrit un mécanisme d'interaction mutuelle entre deux éléments, à la fois sur le plan du type de la structure de qualia résultant mais aussi sur le plan des instanciations de ses rôles. L'exemple prototypique de ce mécanisme est le syntagme faire un gâteau.

Enfin, les représentations lexicales construites dans le cadre du LG sont organisées autour de quatre niveaux de description: la structure argumentale (elle liste et type l'ensemble des paramètres logiques non événementiels, au sens de la logique des prédicats, permettant de représenter le contenu sémantique du lexème étudié), la structure événementielle (elle liste et type l'ensemble des paramètres logiques événementiels nécessaires à la description sémantique du lexème), la structure de qualia qui décrit les quatre facettes ou rôles décrivant le contenu sémantique du lexème et qui permet la construction de sens contextuels.

Enfin, la structure de qualia lie entre eux tous les paramètres des structures argumentale et événementielle) et le type de la structure de qualia dans la structure générale des types du modèle (encore appelé 'Lexical Conceptual Paradigm' (Paradigme Conceptuel Lexical) et noté type_lcp, cette étiquette de type permet de synthétiser l'ensemble des dénotations possibles du lexème étudié). Dans la suite, nous ne décrirons que la structure de qualia ainsi que le type LCP.

\subsection{Les syntagmes à sens différentiel et le modèle LG}

Au cours de ses travaux de doctorat, Pierrette Bouillon a produit une classification des adjectifs en français, et associées à celle-ci, des représentations dans le modèle LG pour deux classes parmi les sept qu'elle a ainsi délimité.

Au vu des critères avancés dans (Bouillon 1997), l'ensemble des adjectifs entrant dans des syntagmes à sens différentiels entrent dans trois classes du point de vue syntaxique :

- classe 1 : les adjectifs sans argument (chaste, prude, sociable, modeste, galant, léger);

- classe 2 : les adjectifs événementiels à deux arguments (facile, difficile, impossible) et (sage, sérieux, honnête, libre). Ces adjectifs admettent plusieurs propriétés distinctives comme l'extraposition il est facile de sortir pour Jean / Sortir est facile pour Jean ; il est sage de la part de Jean de sortir / Sortir est sage de la part de Jean, etc.

Sur le plan sémantique, la seconde classe se subdivise ainsi : les adjectifs (facile, difficile, impossible) s'appliquent à un événement qui est perçu, éventuellement par un individu, comme ADJ ; les adjectifs (sage, sérieux, honnête, libre) de leur côté, s'appliquent à un individu qui a la propriété d'être ADJ, propriété qui se manifeste lorsqu'il exerce une certaine activité ou qu'il accomplit un certain événement. Pour cette raison, cette deuxième subdivision est appelée par Bouillon des adjectifs orientés-agent.

(7) Les adjectifs événementiels reçoivent une représentation telle que (7) et les adjectifs orientés-agent, telle que (8) (Bouillon 1997 : 105). Le rôle télique est choisi pour les adjectifs orientés-agent car il décrit l'activité typique de l'individu par laquelle il manifeste qu'il a la propriété ADJ ; pour les événementiels, le rôle agentif est choisi car il faut que l'événement dit facile par exemple soit d'abord perçu comme tel 
pour pouvoir être ensuite considéré comme ayant la propriété facile, la perception est donc une condition nécessaire en (8).

(8) adjectif $\mathrm{ADJ}$

QUALIA = orienté-agent_lcp

FORMEL = 'qualité ou défaut ADJ de l'individu x co-occurrent avec l'adjectif'

TELIC $=$ 'activité ou action de $\mathrm{x}$ '

(9) adjectif $\mathrm{ADJ}$

$$
\begin{aligned}
\text { QUALIA }= & \text { événementiel_lcp } \\
& \text { FORMEL }=\text { 'propriété ADJ de l'événement e co-occurrent avec l'adjectif' } \\
& \text { AGENTIF }=\text { 'perception de e' }
\end{aligned}
$$

\subsection{Représentation de homme et femme}

Comme nous l'avons vu dans la section (1), la notion d'être sexué est prédominante pour la femme, et celle d'être social, pour l'homme. De telles distinctions pourraient être représentées de la manière suivante en LG.

(10) homme

$$
\begin{aligned}
\text { QUALIA }= & \text { human_individual_male_lcp } \\
& \text { FORMEL }=\text { 'individu' ET 'être humain' ET 'comportement social' } \\
& \text { CONSTITUTIF }=\text { 'sexe mâle, cheveux, etc.' }
\end{aligned}
$$

(11) femme

$$
\begin{aligned}
\text { QUALIA }= & \text { human_individual_female_lcp } \\
& \text { FORMEL }=\text { 'individu' ET 'être humain' ET 'comportement sexuel' } \\
& \text { CONSTITUTIF }=\text { 'sexe femelle, cheveux, etc.' }
\end{aligned}
$$

\subsection{Composition N+Adj et Adj+N dans LG et sens différentiel}

Dans le cadre des travaux (Bouillon 1997), et précisément concernant la composition $\mathrm{N}+\mathrm{Adj}$ ou Adj+N, Bouillon ne remet pas en cause le fait que la position postnominale est non marquée et qu'à ce titre, la composition nom adjectif n'est pas sensée modifier la dénotation du nom. En revanche, concernant la position prénominale, deux cas se présentent.

- Si l'adjectif peut figurer avant et après le nom et que l'on constate une variation sémantique, ce phénomène sera représenté par le fait qu'en position postnominale, l'adjectif n'a accès qu'à la tête de la structure de qualia du nom, c'est à dire le rôle formel, et qu'en position prénominale, il aura accès à l'ensemble des rôles de la qualia du nom.

- Si l'adjectif peut figurer uniquement après le nom, ou bien si quelle que soit sa position, le contenu sémantique du syntagme reste stable, alors, quelle que soit la position de l'adjectif, il a accès à tous les rôles de la qualia du nom.

(12) Dans le cas des adjectifs que nous étudions, hors expressions figées, on se trouve plutôt dans le deuxième cas, notamment avec honnête qui déclenche le sens différentiel avec femme aussi bien en position prénominale que postnominale. 
Enfin, si l'on combine l'ensemble des observations faites jusqu'à maintenant ainsi que les principes de formalisation proposés, on aboutit au mécanisme suivant qui explique en partie ces interprétations différentielles trouvées en corpus. Avec honnête ou sage, qui sont des adjectifs orientés-agent modifiant un individu, la combinaison $\mathrm{N}+\mathrm{ADJ}$ produira naturellement une honnête femme sexuellement interprétée : la contrainte pour l'adjectif de modifier un individu est satisfaite par la première partie de l'instanciation du rôle formel de femme 'individu' et l'interprétation voulue est obtenue grâce à la mention du comportement sexuel dans le rôle formelle de l'entrée de femme. Avec facile, la combinaison N+Adj pose problème dans la mesure où le nom femme ne dénote pas un événement. Dans ce cas, le mécanisme de la coercion va permettre de construire un type événementiel pour la structure de qualia du syntagme et à cette fin, l'adjectif facile se combinera avec la seule représentation d'un événement que comporte la structure de qualia de femme, à savoir son comportement sexuel. Enfin, lorsqu'aucune interprétation différentielle n'est constatée (difficile, impossible, chaste, pudique, modeste), il nous faudra déterminer les moyens de représenter les différents éléments caractéristiques : caractère négatif de l'adjectif, l'adjectif est socialement marqué (sociable) ou sexuellement marqué (prude, chaste), l'adjectif est événementiel ou sans argument.

\section{Conclusion}

Essayer d'expliquer le sens différentiel en termes formels contredit la prise de position la plus courante, dans le domaine de la linguistique, qui prend pour acquis un lexique qui ne peut contenir la connotation. Cependant, nous basant sur des concepts fondamentaux tels que les définitions de "homme" et de "femme" qui mettent en évidence l'importance des axiomes socialement normés, nous avons proposé d'identifier la partie de la signification expliquée par la sémantique lexicale et celle par les connaissances encyclopédiques et sociologiques. La formalisation proposée dans le cadre du lexique génératif nous a ainsi permis de formuler une hypothèse sur les qualia de "homme » et de «femme » afin de rendre compte des aspects de la signification qui sont dépendants d'un contexte socio-culturel plus large.

\section{Références bibliographiques}

Armengaud F. (1999). Introduction. Nouvelles Questions Féministes, 20(1) : 2-7. 1999. 3.

Baider, F. et A.-M. Brousseau (1999). Qualia et coercion sémantique: pour une représentation formelle de la connotation. Annual meeting of the Canadian Linguistic Association. Congrès des Sociétés savantes, Sherbrooke, Canada.

Baider F. et E. Jacquey (2007). La place du genre dans les bases de données multilingues : le cas d'EuroWordNet. Nouvelles Questions Féministes 26 (3)10. 57-69.

Bailly R. (1947). Dictionnaire des synonymes de la langue française. Paris : Larousse.

Beaujot, J. P. et M. A. Lehmann (1979). Langue, idéologie, dictionnaire: le discours sur FEMME et FILLE dans le Petit Larousse Illustré de 1906 à 1978. Bulletin du centre d'Analyse du discours de l'Université de Lille. Villeneuve d'Ascq : Publications de l'Université de Lille 3 (1976-1978). 1-175.

Benhamou, S. (1986). Analyse dictionnairique de femme et de homme. Cahiers de Lexicologie, vol XLVIII, I. $27-67$.

Bertaud de Chazaud, H. (1987 [1983]). Dictionnaire des synonymes. Paris: Le Robert

Bouchard, D. (1995). Order of Adjectives and Structure of the Noun Phrase. Communication faite à l'Université de Toronto, Département de linguistique, ms.

Bouillon P. (1996). Mental state adjectives: the perspective of Generative Lexicon. International Conference On Computational Linguistics, Proceedings of the $16^{\text {th }}$ conference on Computational linguistics. Vol.1, Copenhagen, Danemark. 143-148.

Bouillon P. (1998). Polymorphie et sémantique lexicale : le cas des adjectifs. Lille : Septentrion Presse Universitaires.

Cameron D. (édit.). Feminist Critique of Language. London: Routledge. 134-147. 
Copestake, A. \& T. Briscoe (1996). Semi-Productive Polysemy and Sense Extension. Lexical Semantics. The Problem of Polysemy. Pustejovsky, James \& B. Boguraev (eds). Oxford: Clarendon Press.

Cruse, A. (1986). Lexical Semantics. Cambridge: Cambridge University Press.

Grzesitchak, M ; E. Jacquey et F. Baider. (2008). Annotation sémantique : profilage textuel et lexical. Présenté à au colloque Lexicographie et informatique : bilan et perspective, Université Nancy 2. ATILF- CNRS, ms.

Eakins, B et R. Gene Eakins (1978). Sex Differences in Human Communication. Boston: Houghton Mifflin.

Fellbaum, Ch. (1998). WordNet, an electronic lexical database. Cambridge, Massachusetts: MIT Press..

Houdebine-Gravaud, A-M (1999). Femmes/langues/féminisation: Une expérience de politique linguistique en France. Nouvelles Questions Féministes, 20 (1). 23-53.

Jacquey E.et Seddah D. 2002. Conceptualization of a lexical information system. Nancy: /TKE2002/.,.

Jacquey E. (2005). Ambiguïté lexicale et quantification : une modélisation de la polysémie logique. Interpréter en contexte. Francis Corblin et Claire Gardent (eds.). Hermès. 107 - 143.

Khaznadar, E. (2002). Féminin à la française. Paris : L'Harmattan.

Lakoff, R. (1975). Language and Woman's place. New York: Harper \& Row.

Michard, C. (2000). Sexe en linguistique - Sémantique ou zoologie. Paris : L'Harmattan.

Miller, C. et K. Swift (1976). Words and Women. Garden City, NewYork: Anchor Press.

Pustejovsky, J. (1995).The Generative Lexicon. Cambridge (MA)/London(UK): The MIT Press.

Rastier, F. (1987). Sémantique interprétative. Paris : Presses Universitaires de France.

Schultz, M. (1990 [1975]). The Semantic derogation of woman. The Feminist Critique of Language. Cameron D. (édit.). London : Routledge. 134-147.

Scullen, M-E (2003). Les dictionnaires français : un lieu privilégié du sexisme ? Cahiers de Lexicologie 83. 131-153.

Yaguello, M. (1992 [1978]). Les Mots et les femmes. Paris : Payot.

${ }^{1}$ Le TLFi (Trésor de la Langue Française Informatisé) est consultable en ligne à http://www.atilf.fr/tlfi.htm.

${ }^{2}$ Pour une analyse plus détaillée de ces hiérarchies voir Baider et Jacquey (2007).

${ }^{3}$ Les mots en corrélation au terme efféminé, en majorité de nuance péjorative (lâche, faible), sexuelle (sensuel, languissant) ou les deux (vicieux).

${ }^{4}$ Nos données proviennent et décrivent le français de France. Tous les sens des adjectifs discutés dans ce travail sont les sens les plus fréquents trouvés dans FRANTEXT. Le mot interprétation fait référence au sens spontané que les Francophones donnent aux données trouvées en corpus, que ce soit les données sur Frantext, dans le TLFi ou dans Google.

${ }^{5}$ Dans le TLFi : En parlant d'une femme «Dont la conduite est conforme à une norme sexuelle (liée à un statut social) $»$.

${ }^{6}$ Ainsi la citation suivante dans FRANTEXT: Justement comme j'étois au coin de la rue, je vis mon frère entrer dans un lieu où je savois qu'il ne demeuroit que des filles d'une vertu facile. Je crûs d'abord m'être trompé (Chasles, Illustres françaises, 1959 [1713] : 418). 